$$
\begin{aligned}
& \frac{\text { A "Figure-of-Merit" Approach to Extraterrestrial }}{\text { Kesource Utilization }} \\
& \text { Kepartment of Aerospace and Mechanical Engineering } \\
& \text { The University of Arizona }
\end{aligned}
$$

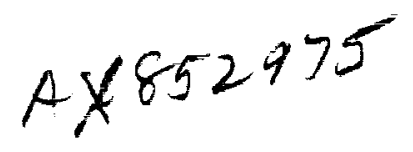

Abstract

A concept is developed for interrelated optimizations in space missions that utilize extraterrestrial resources. It is shown that isolated (component) optimizations may not result in the best mission. Overall economics in the broadest sense must include the "costs" of transportation, storage, continuous monitoring, and corrective actions in situ. When all of these needs are quantitatively considered, it is shown that substantial benefits can be had through less-than-the-best propellants, propellant combinations, propulsion hardware, and, actually, some waste in the traditional sense. One ready example is the possibility of discarding hydrogen produced extraterrestrially by water splitting and using only the oxygen to burn storable fuels. The gains in refrigeration and leak-proof equipment mass (elimination) outweigh the loss in specific impulse.

After a brief discussion of this concept, the synthesis of the four major components of any future space mission is developed. The four components are: orbital mechanics of the transportation; performance of the rocket motors; support systems that include power, thermal and process controls, and instruments; and in-situ resource utilization plant equipment. State-of-the-art numbers are used for the components' performances; each is studied in depth elsewhere, but those studies are beyond the scope of this paper, whose main aim is the development of the concept of a figure-of-merit for the mission. One specific example is used to illustrate the new concept; this is the Mars Sample Return mission. At this time, a popular spreadsheet is used to quantitatively indicate the interdependent nature of the mission optimization. Future prospects are outlined that promise great economy through extraterrestrial resource utilization and a technique for quickly evaluating the same.

Introduction

The remarkable potential for cost reduction in space missions that use extraterrestrial resources, as contrasted with more traditional missions that depend exclusively on all-Earth-transported resources, has been amply discussed elsewhere (Ash and Cuda 1984; Ash et al. 1978, 1982; Carroll 1983; French et al. 1985; Frisbee and Jones 1983; Ramohalli et al. 1987a, 1987b, 1989; (Presidential) National Commission on Space 1986). Every pound, or kilogram, that can be saved in the initial liftoff mass from Earth can pay back several pounds, or kilograms, because of the leverage effect 
of the mass ratio equation. In this regard, it is obvious that we should specifically target those fractions of the liftoff mass that have the maximum impact on the mission. Even a cursory glance at the state-of-the-art spacecraft indicates that a very large fraction of the overall mass is the propellant mass. Thus begun the studies that explored the possibilities of manufacturing propellants in situ on extraterrestrial bodies to "refuel" out there, instead of from Earth-transported precious propellants. These ISPP studies concluded that it is definitely advantageous to manufacture propellants extraterrestrially. The pioneering paper by Ash et al. (1978) showed the first technical aspects of such an ISPP mission. More recent studies (Ramohalli et al. 1987a, 1987b, 1989) not only looked at specific missions, but also explored the general class of extraterrestrial resources instead of just resources to be used as propellants. These results were sufficiently promising for NASA to establish a Center devoted to utilization of extraterrestrial resources. In addition to the all-important propellants, structural materials, shield materials, and other useful materials are being seriously considered. The fundamental goal is to render large-scale space operations economically feasible without depending upon a fleet of dedicated heavy-lift launch vehicles and upon Earthtransported repair, relief, and support supplies. It is also of utmost importance to evolve a long-range ecologically acceptable plan to minimize contamination, and subsequent cleanup, of space.

After this recognition of the significant merits of extraterrestrial resource utilization, some of the more traditional thoughts on space missions may have to be re-examined. For example, many of the components and systems that are used almost routinely for their superior performance may not be the best ones in light of the overall mission impact. Simple examples will illustrate the point. Oxygen-hydrogen rockets have been used almost exclusively because of their excellent specific impulse, years of accumulated design experience, contingency margins, and reliability. In fact, use of the $\mathrm{LOX}-\mathrm{H}_{2}$ system for primary, and even secondary, propulsion seems beyond question for future space missions. And yet when one considers the cryogenics, refrigeration, leaks, long-term material compatibility, power needs in simple storage, and safety of remote operations, several important questions arise. It is necessary to consider the full support equipment, too, in space missions. When one adds these support masses to the primary LOX- $\mathrm{H}_{2}$ propulsion system, the desirability picture changes. It may be better to utilize a propellant system that is far less demanding in terms of refrigeration, leaks, and other related aspects, even if the specific impulse performance is inferior. The point is that what we lose in specific impulse can be more than made up in the Earth-transported mass. Some of the storable propellant combinations are seen to offer several advantages. 
Expanding on these simple thoughts, we next consider several other aspects, such as long-term reliability, repairability, ease of resupply, and multiple use of components (e.g., Can an empty propellant tank become the chamber for the next-stage rocket? Can the TVC injector for the first stage become the main propellant injector for the third stage?). Also, what are the implications of using some of the unconventional propellants in rocket hardware? Will injector nozzle buildup and blockage become a serious problem? Will some of the highly solids-laden flow cause excessive erosion of the nozzle throats? With the less energetic propellants, can we use a simple nozzle and not worry about replacing critical nozzle throats? What is the overall gain to the mission?

By now, it is obvious that future space missions, especially those designed to utilize extraterrestrial resources, will have to be designed from an overall mission advantage point of view rather than in a simple component-optimized fashion. This may seem simple enough, but we must evolve a quantitative engineering design methodology that reflects this new design philosophy. We term this the figure-ofmerit (FoM) approach. Although it may seem like an over-simplification, one number may be useful, purely for the purposes of initial screening of a myriad of concepts, in characterizing the overall merits of a space mission The figure-of-merit considers the specific impulse, mass ratio, reliability, inverse risk, repairability, ease of autonomous controls, scalability, and adaptability. We recognize that this is only the first step; more involved considerations will be added later. Here, the idea is to introduce a new methodology that is quantitative and considers the known engineering facts and technologies for future space missions.

The next section gives a brief overview of the components of the space missions of interest. These missions are largely the recommendations of the Sally Ride report and the Space Commission report. To render some of these thoughts more concrete, it is important to consider some specific missions. We have chosen one of the most popular missions of the next century, namely, Mars Sample Return (MSR). An advantage of this mission is that it has been extensively studied both in the USA and the USSR, including some joint-effort possibilities. Thus, it is easy to see the advantages of the FoM approach over the conventional approach. The basic mission "ground rules" are mentioned in order to provide a meaningful basis for comparisons. Whenever hard numbers are not readily available, the best estimate has been used based upon conversations with experts; it is an easy matter to change these numbers as they become more exact.

The heart of this paper considers the development of a quantitative, interrelated, color-coded program that enables the FoM to. be readily examined for all mission 
variations. This was first accomplished through the use of simple manual entry into one of the popular spreadsheets (Lotus 1-2-3). After checking for consistency and simple verifications, the program is now being upgraded to use one of the expert systems. While other researchers may have taken similar approaches to this interrelated "spreadsheet" development, our main contribution has been the detailed quantitative considerations of the ISPP itself. Since the ISPP modules represent the most critically important components of the entire mission, it is very important to treat them in detail. The results indicate the definite advantages of this FoM approach.

It is concluded that the FoM approach may provide a powerful and yet easy and simple tool to design future space missions that can introduce great economy (and ecology) through the utilization of local planetary (extraterrestrial) resources.

\section{The Concept of "Figure-of-Merit"}

It is very important to have a clear understanding of what is meant by the figureof-merit. The FoM could be different for different missions and should be different for different priorities. The simplest definition appears to be simply the payload ratio as the most easily understood form of FoM. In some circles, the specific impulse $\left(I_{s p}\right)$ is good enough as the FoM. In the context of the more complex missions that we need in order to utilize extraterrestrial resources, several possible definitions are indicated in Table 4.1.

Table 4.1. Possible definitions of a figure-of-merit.

1. $\frac{m_{p l}}{m_{\text {launch }}}=\frac{m_{\text {payload }}}{m_{\text {launch }}}$

2. Total Life-Cycle Cost of This Mission

3. State-of-the-Art Launch Cost of This Payload

4. Mass of the Sample Returned to LEO

5. Total Useful Mass

6. Any of the above, modified by factors for Reliability $\left(R_{e l}\right)$, Repairability $\left(R_{e p}\right)$, Inverse Risk (1/Risk), ...

Note: Definition 1 will not consider the shuttle external tank in the numerator, but Definition 5 may include the ET in the numerator if it can be usefully employed in the space operation, such as ISRU. 
For the purposes of introducing the concept of the FoM approach in extraterrestrial resource utilization and for the purposes of this paper, we have chosen definition number four (4), modified by factors for reliability, repairability, and inverse risk (Table 4.2); also, a factor of $10^{4}$ is used as a multiplier for reasons of convenience. We recognize the limitations of the above definition. For example, it gives no indication of the relative ease, or difficulty, of getting to LEO in the first place. It also gives no indication of the ease, or difficulty, of assembling the spacecraft in LEO to be launched towards its destination. If the main intention of the mission is an extended duration settlement on Mars, the return of a small sample to LEO is no indication of the FoM. The main point is to start with an FoM that is easily modified subsequently while retaining the meaning and spirit of the quantitative interpretation and the flexibility to accommodate the modifications. Our present choice of the FoM definition possesses that capability. Thus, for the purposes of this initial introduction,

$$
\text { FOM }=\frac{m_{\text {pl.returned }}}{m_{\text {launch.LEO }}} R_{\text {el }} \cdot R_{\text {epr }} \cdot(1 / \text { Risk }) \cdot 10^{4} .
$$

In this specific example of MSR, the assembled spacecraft is launched from LEO on a Hohmann ellipse. The craft is made to enter a circular Mars parking orbit (MPO) approximately $200 \mathrm{~km}$ above the Martian surface. The orbiting craft is decelerated by aerobreaking (through the thin atmosphere of $\mathrm{CO}_{2}$ ). Soft landing is achieved through retrorockets. The craft spends several months on Mars, until the time is right for a minimum energy transfer and travel to Earth. The Mars ascent vehicle (MAV) is propelled to the MPO, from which the vehicle is sent on a Hohmann ellipse towards Earth. Upon approaching Earth, the craft is captured in a highly elliptic orbit around Earth, instead of the more energy-consuming circular LEO. The design, configuration, and operation of the MAV is critical, and the entire mission FoM is extremely sensitive to changes in the MAV design. The energy requirement calculations ( $\left.\Delta v^{\prime} s\right)$ are straightforward and, after verification with two reliable sources (Irving and Blum 1959, Bursard and deLauer 1965), we used the numbers given in Table 4.3.

Table 4.2. The modifier factors. ${ }^{a}$

\begin{tabular}{lllllll}
\hline & \multicolumn{5}{c}{ Cases } \\
\cline { 2 - 6 } & 1 & $2 a$ & $2 b$ & 3 & 4 \\
\hline $\begin{array}{l}\text { Risk/Inverse Risk and } \\
\text { Reliability \& Repairability }\end{array}$ & 0.9 & 0.7 & 0.7 & 0.6 & 0.2 \\
\hline
\end{tabular}

a These numbers represent the total effects of all the factors. They have not been reviewed individually as yet. 
Table 4.3. The $\Delta v$ 's for the Mars Sample Return mission.

Physical Constants
Radial distance from body to Sun, Earth-Sun ref: $\begin{aligned} & \text { Earth }=1.00000 E+00 \\ & \text { Mars }=1.52400 E+00\end{aligned}$
Radial distance from body to Sun [m]: $\begin{aligned} & \text { Earth }=1.49600 E+11 \\ & \text { Mars }=2.27990 E+11\end{aligned}$
GZERO [m/s] $-9.81000 E+00 \quad$

Propulsive Velocity Requirements [m/s]

LEO to Hohmann towards Mars (Stage I) $=1.90000 E+03$

LEO to Hohmann towards Mars (Stage II) $=1.90000 E+03$

Hohmann to Mars parking orbit (MPO), $200 \mathrm{~km}$ (Stage I includes $0.5 \mathrm{~km} / \mathrm{s}$ midcourse correction) $=1.85000 E+03$

Hohmann to Mars parking orbit, $200 \mathrm{~km}$ (Stage I) $=1.35000 \mathrm{E}+03$

MPO to Mars soft landing $=1.00000 E+03$

Mars ascent vehicle to MPO (Stage $I$ ) $=1.75000 E+03$

Mars ascent vehicle to MPO (Stage II) $=1.75000 \mathrm{E}+03$

MPO to Hohmann towards Earth (Stage I) $=1.35000 E+03$

MPO to Hohmann towards Earth (Stage II) $=1.35000 E+03$

Hohmann to highly eccentric Earth orbit (includes $0.5 \mathrm{~km} / \mathrm{s}$ midcourse correction) = $1.50000 \mathrm{E}+03$

Four different scenarios, to accomplish an identical MSR, are considered in detail. The first is the base line (case 1) and involves state-of-the-art, Earth-transported resources; LOX/H $\mathrm{H}_{2}$ propulsion; and the associated cryogenic storage both en route and on Mars. Variation 1 (case 2) considers Earth-transported resources, but employs storable propellants in order to clearly reveal the influence of refrigeration as distinct from the influences of in-situ resource utilization (ISRU); here, a fairly routine oxidizer $\left(\mathrm{N}_{2} \mathrm{O}_{4}\right)$ and a slightly unconventional oxidizer in space missions $\left(\mathrm{H}_{2} \mathrm{O}_{2}\right)$ are considered as cases $2 a$ and $2 b$. Variation 2 (case 3 ) considers Earth-transported fuel (hydrocarbon) and the production of the oxidizer from the Martian atmosphere; the ISRU equipment to produce oxygen on Mars is Earth-transported. Variation 3 (case 4) considers only ISRU propellants; oxygen is manufactured on Mars through Earth-transported equipment. In variations 2 and 3 , the Earth-transported ISRU equipment is left behind on Mars and is not part of the MAV. In variation 3, the fuel is a hydrocarbon derived from the spent case of the Mars lander vehicle (Kevlar or Nylon). The specific impulse of the MAV is highest in the base line case and lowest in variation 3. The specific cases considered 
in this paper are shown in Table 4.4. It is a simple matter to consider various other combinations of fuel/oxidizer, chamber pressures, nozzle expansion ratios, rocket chamber and propellant container cases, and all related equipment.

Table 4.4. Comparative study.

\begin{tabular}{|c|c|}
\hline $\begin{array}{c}\text { Base Line } \\
\text { (case 1) }\end{array}$ & All Earth-transported resources, $\left[\mathrm{OX} / \mathrm{H}_{2}\right.$ propulsion, cryogenic storage \\
\hline $\begin{array}{l}\text { Variation } 1 \\
\text { (case } 2 a ; b)\end{array}$ & $\begin{array}{l}\text { All Earth-transported resources, storable propellants }\left(\mathrm{CH}_{4} / \mathrm{N}_{2} \mathrm{O}_{4} \text {; }\right. \\
\left.\mathrm{CH}_{4} / \mathrm{H}_{2} \mathrm{O}_{2}\right)\end{array}$ \\
\hline $\begin{array}{c}\text { Variation } 2 \\
\text { (case 3) }\end{array}$ & $\begin{array}{l}\text { Earth-transported fuel (HC), in-situ resource utilization (ISRU) of } \\
\text { from atmosphere }\left(\mathrm{CH}_{4} / \mathrm{LOX}\right)\end{array}$ \\
\hline $\begin{array}{c}\text { Variation } 3 \\
\text { (case 4) }\end{array}$ & All \\
\hline
\end{tabular}

\section{A Brief Description of Components}

The overall system to accomplish the mission depends upon the performance of the individual components. It is important to understand the nature of the components that comprise the integrated system. Such an understanding also enables us to change the components, almost in a modular fashion, to see the influences of variations and the sensitivity to changes, and to incorporate advances in technology. It is the specific intention of this FOM approach to retain the flexibility to incorporate any change in any component in a clearly understandable manner. Thus, this approach is distinctly different from complex programs and software "packages" that frequently fail to reveal the component influences individually. The fundamental essence of this FoM approach is shown in Figure 4.1. The components are fairly straightforward and have been discussed in the literature. For the purposes of this paper, we give only a brief description of the components that make up the FoM system.

The Mission. As was stated earlier, the mission we chose as a case study is the Mars Sample Return. The high-thrust propulsion technique is employed. The incremental velocities needed are broken up into different stages, as shown in Table 4.3. Some midcourse corrections, $\Delta v$ 's needed to account for the noncircular nature of orbits of the Earth and Mars (around the Sun) and the non-coplanar nature of the two orbits, are all taken as incidentals and amount to a total of approximately 1 to $2 \mathrm{~km} / \mathrm{s}$. The return journey is on a Hohmann ellipse, but the Earth capture is in an energyefficient, highly elliptical orbit rather than a circular orbit. The main variations are not so much in the value of the $\Delta v$ 's necessary, but in the way one could choose to split 


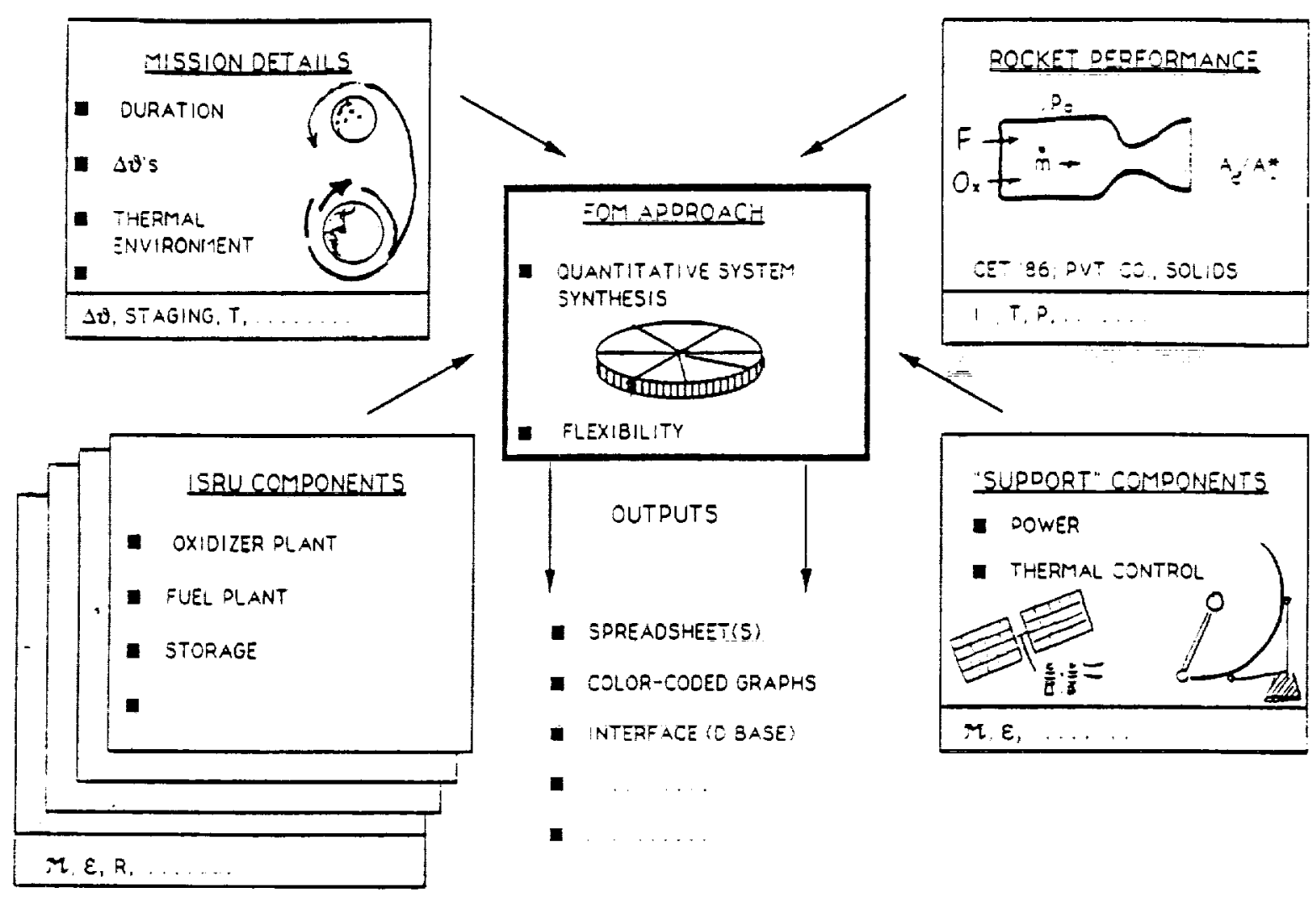

Figure 4.1 The essence of the figure-of-merit concept.

the stages. Our choice is by no means the best, but is consistently used, so the comparisons are meaningful.

Rocket Performance. The performance of the rocket(s) at various stages of the mission are needed in order to calculate the mass ratios. The main program used here is CET86, which is an improvement over the highly popular CEC72 program, from NASA Lewis Research Center. The PC version of this program was obtained from the University of Minnesota, and the results were verified with the mainframe version of the program at the University of Arizona. Another version of this program was obtained from a private company. This version has the specific capability of handling large fractions of charged species, or plasmas. Yet another program has been independently developed by a private company in California for the specific purpose of handling large fractions of solid particles in the products. This program is also installed on a PC (with a 386 board) at the University of Arizona. Thus, two versions of the NASA Lewis program, a variation with plasma capability enchancement, and an independent thermochemical program were used. After satisfactory verifications, the CET86 output was used as the input in this FoM study. More than 30,000 data sets were generated and covered various fuels, oxidizers, fuel/oxidizer ratios, chamber pressures, and 
expansion ratios in the nozzle. Both equilibrium and frozen cases were run, and usually the mean was taken as the reliable number. The output includes the usual matrix of specific impulse, temperatures, and species concentrations, along with a myriad of parameters. Unconventional propellants and combinations were specifically studied, as were the more conventional ones. $\mathrm{LOX} / \mathrm{H}_{2}$ formed the standard basis for comparisons. Its performance is illustrated in Figure 4.2. Some of the unconventional combinations involved highly fuel-rich and highly oxidizer-rich cases; some unconventional fuels included Nylon and Kevlar burned with oxygen; some unconventional oxidizers included hydrogen peroxide. These unconventional propellant studies are intrinsically important, and are important to space missions, but the details are beyond the scope of this paper. They are the subject of a Master's thesis (Rascon 1989), the extensive calculations of which will be published shortly.

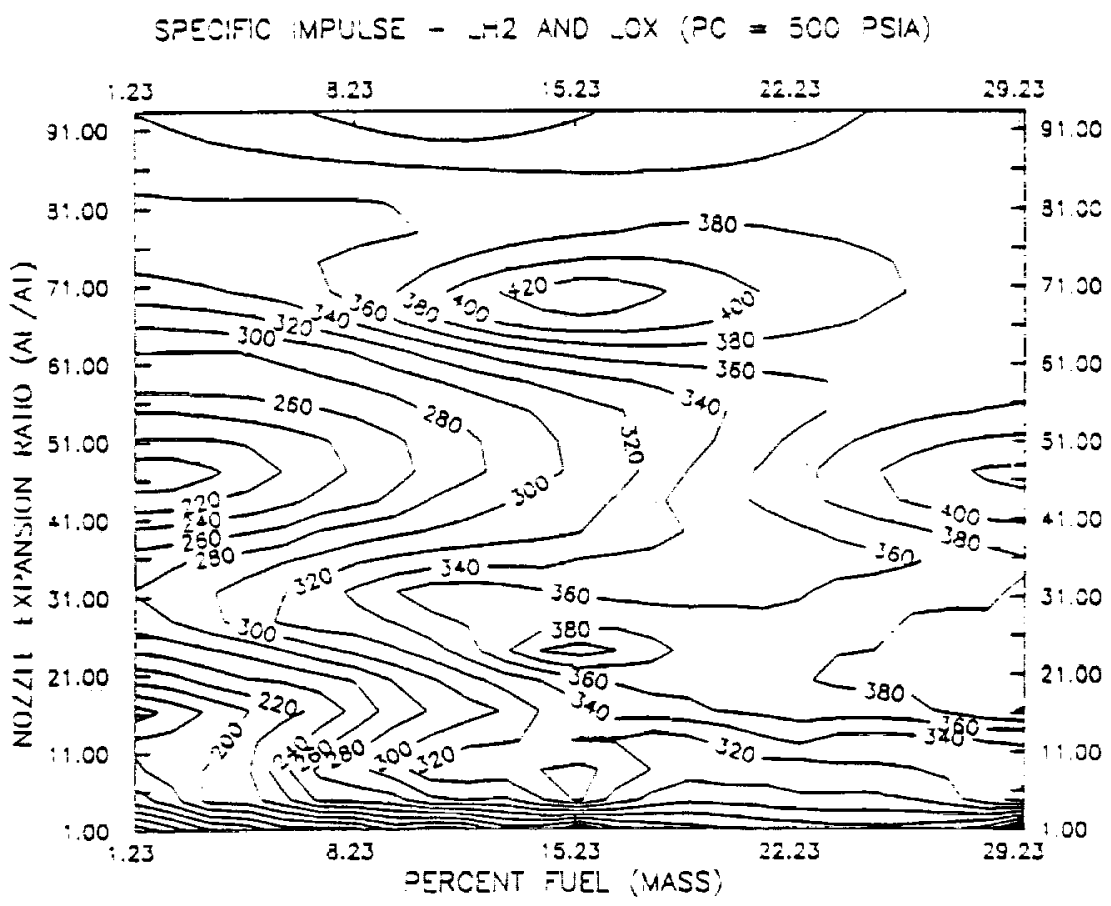

Figure 4.2 Typical performance contour for $\mathrm{LH}_{2} / \mathrm{LOX}$.

The "Support" Components. These include the power, thermal control, radiation shield, pumping, storage, and monitoring equipment. Again, these are very important to the FoM calculations, but do not form the mainstream of our discussions here. We have used the state-of-the-art numbers for nuclear, radioisotopic, solar photovoltaic, solar dynamic, and solar thermal power sources. The sources of these are shown in Table 
4.5. These "support" components are likely to undergo rapid advances in the next few years and will ultimately translate into lower masses and costs.

Table 4.5. The "support" energy data.

\begin{tabular}{lr}
\hline RTG & $333 \mathrm{~kg} / \mathrm{kW}$ \\
Nuclear & $340 \mathrm{~kg} / \mathrm{kW}_{\mathrm{e}}$ \\
Solar PV & $300 \mathrm{~kg} / \mathrm{kW}$ \\
Solar Dynamic & $100 \mathrm{~kg} / \mathrm{kW}_{\mathrm{e}}$ \\
\hline
\end{tabular}

The In-Situ Resource Utilization Components. The in-situ resource utilization (ISRU) components represent the very core of the present study to the extent that the ISRU influences upon space missions are brought out by this FoM approach. The NASA Center at the University of Arizona is devoted to the development of ISRU. Various schemes are being considered for the manufacture of propellants and other useful materials from extraterrestrial resources. Associated with these are innovative concepts for energy utilization, separation of species in microgravity, reactor designs, and storage. The details, as of early 1989, are available in the University of Arizona, NASA Space Enginering Research Center "Annual Progress Report 1988-89." The idea of ISRU is relatively new, and the available studies have taken a somewhat traditional(!) approach. This statement is not as paradoxical as it may seem at first glance. The advantages of ISRU have been well recognized indeed; the approaches toward ISRU have involved ideas of transporting terrestrially used processing plants to extraterrestrial sites in order to manufacture useful substances "out there." Some innovation, a little high technology, some reductions in mass, and smaller safety margins have been the recipe of such proposed ISRU missions. At the NASA SERC of the University of Arizona, a fundamentally different approach is being taken. Here, the aim is to design the ISRU systems as one would design them from local resources, using the information bank from terrestrial and previous space missions. The details used in this paper are shown in Table 4.6. The numbers and technologies will certainly change and will be incorporated in future calculations.

Table 4.6. The ISRU plant data for LOX production on Mars.

$70 \mathrm{~kg}(\mathrm{Zr}$ cells)

$10 \mathrm{~kg} /$ day

$425 \mathrm{~kg}$ support mass 
The Development of the Interdependent Matrix

The purpose of this section is to develop the fundamentals of the interdependent matrix that can evaluate the influences of various components upon the overall system. Mathematically, the problem can be formulated as an optimization problem with constraints. The use of Lagrange multipliers may seem natural. When one considers that there are at least twenty constraints at a time and that the nature of the mass equation is highly nonlinear (exponential), the use of analytical methods may not be straightforward. There are many modern techniques that could be used for such a problem. At the present time, we have used a popular spreadsheet (Lotus 1-2-3) to represent the interdependent matrix. The actual technique of quantitatively interrelating the variables and calculating the optimum is not critically important for the purposes of this paper, although the evolution of the optimum technique could be as important as the evolution of the optimum itself in terms of the economy of arriving at the solution. The use of this spreadsheet has the advantages of simplicity, popularity and ease of use at various locations, ready color graphing capability, and the ease of interfacing with other software. There is certainly room for improvement, and that is the subject of the graduate thesis of the junior author.

The Evolution of the Spreadsheet

The spreadsheet was used to build the relationship between the initial mass and final mass in a single-stage rocket. The usual approach is to select a propellant and make an estimate of the structural mass. In this study, the structural mass was separated into different system masses, starting with the most general case. The different options included the aeroshell, nozzle, guidance and control, fuel tankage, oxidizer tankage, and a structural mass (pumps, valves, lines, etc.). The refrigeration needs of the propellant were then addressed. An interesting problem arose in the evaluation of the refrigeration needs. The propellant and the refrigeration masses are dependent upon each other. In transit, the dominant effect was assumed to be the solar radiation introducing a heat flux. The spreadsheet was used to solve the equations without attempting to linearize the highly nonlinear expression. The result is the solution of a cubic. Each stage then served as input for the previous stage. The masses for each category were then summed and the FoM was calculated for each case.

Sample Results

These are shown in Table 4.7 and Figures 4.3 and 4.4. The liquid oxygen needs refrigeration on Mars. The heat transfer is taken to be a combination of natural 
Table 4.7. Summary of masses from low Earth orbit $(\mathrm{kg})$ and figures of merit.

\begin{tabular}{|c|c|c|c|c|c|}
\hline Masses & Case 1 & Case 2a & Case 2b & Case 3 & Case 4 \\
\hline $\begin{array}{l}\text { Aeroshell } \\
\text { Fuel }\end{array}$ & $\begin{array}{l}2.48 E+04 \\
4.74 E+05\end{array}$ & $\begin{array}{l}5.86 \mathrm{E}+01 \\
4.72 \mathrm{E}+03\end{array}$ & $\begin{array}{l}6.16 \mathrm{E}+01 \\
9.44 \mathrm{E}+03\end{array}$ & $\begin{array}{l}2.56 \mathrm{E}+01 \\
1.52 \mathrm{E}+03\end{array}$ & $\begin{array}{l}1.01 E+03 \\
1.62 E+04\end{array}$ \\
\hline Fuel leaked & $2.12 E+04$ & $\overline{-}$ & $\overline{-}$ & - & $\overline{-}=$ \\
\hline Fuel tanks & $6.63 E+04$ & $7.73 E+01$ & 1.57E+02 & $2.39 E+01$ & $2.76 \mathrm{E}+02$ \\
\hline Guidance and control & $6.63 \mathrm{E}+05$ & $4.04 E+03$ & 4.37E+03 & $1.06 \mathrm{E}+03$ & 1.17E+04 \\
\hline Nozzle & $6.63 E+05$ & $4.04 E+03$ & 4.37E+03 & $1.06 E+03$ & $1.17 E+04$ \\
\hline Nylon factory & $-\overline{-}$ & - & - & $\overline{-}$ & $1.42 E+02$ \\
\hline Oxidizer & $3.79 E+06$ & $2.30 E+04$ & $2.01 E+04$ & $6.04 E+03$ & $6.50 \mathrm{E}+04$ \\
\hline LOX refinery & $\overline{-}$ & - & - & $1.84 E+01$ & $3.59 E+01$ \\
\hline Oxidizer leaked & $1.69 \mathrm{E}+05$ & - & - & $2.30 E+02$ & $2.49 E+03$ \\
\hline Oxidizer tanks & $2.65 \mathrm{E}+05$ & $2.42 E+03$ & $2.62 E+03$ & $4.26 E+02$ & $4.66 E+03$ \\
\hline Refrigeration (fuel) & $4.20 \mathrm{E}+05$ & - & - & - & - \\
\hline Rover & $1.50 E+02$ & $1.50 \mathrm{E}+02$ & $1.50 E+02$ & $1.50 E+02$ & $1.50 E+02$ \\
\hline Refrigeration (oxidizer) & $4.42 E+04$ & - & - & - & - \\
\hline Structural & $2.65 E+05$ & $2.02 E+03$ & $2.62 E+03$ & $4.26 E+02$ & $4.66 E+03$ \\
\hline Sample & $1.00 E+00$ & $1.00 \mathrm{E}+00$ & $1.00 E+00$ & $1.00 E+00$ & $1.00 E+00$ \\
\hline Sample support & $1.00 E+00$ & $1.00 E+00$ & $1.00 E+00$ & $1.00 E+00$ & $1.00 E+00$ \\
\hline Total & $6.86 \mathrm{E}+06$ & $4.05 E+04$ & $4.39 E+04$ & $1.10 E+04$ & $1.18 E+05$ \\
\hline $\begin{array}{l}\text { Modification factor } \\
\left(R_{e l}, R_{e p} \text {, inverse risk, ... }\right)\end{array}$ & $9.00 E-01$ & $7.00 E-01$ & $8.00 E-01$ & $5.90 E-01$ & $1.94 E-03$ \\
\hline Figure-of-merit (FoM) & $2.62 E-03$ & $3.46 \mathrm{E}-01$ & $3.65 E-01$ & $1.07 E+00$ & $3.28 \mathrm{E}-02$ \\
\hline
\end{tabular}

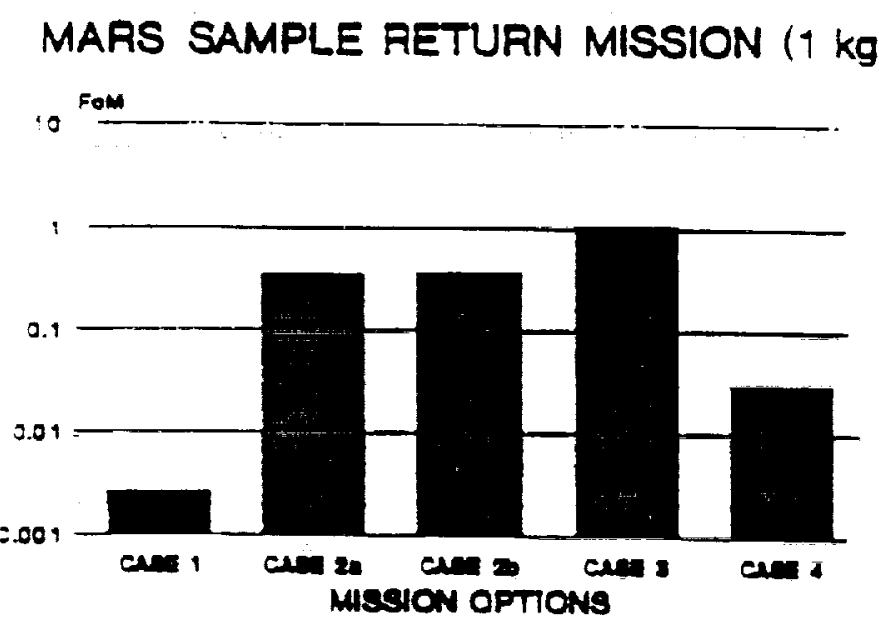

Figure 4.3 Figure-of-merit results. 


\section{COLOR PHOTOGRAPH}

\section{IV-13}

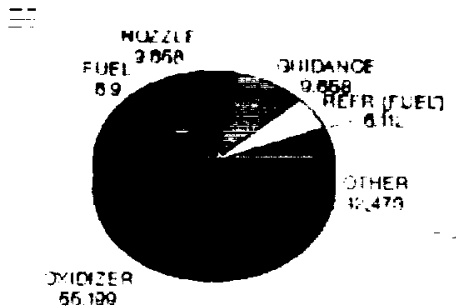

Case 1. All-earth-carried LOX/ $\mathrm{H}_{2}$ (Mass at LEO $=6860$ tonnes)

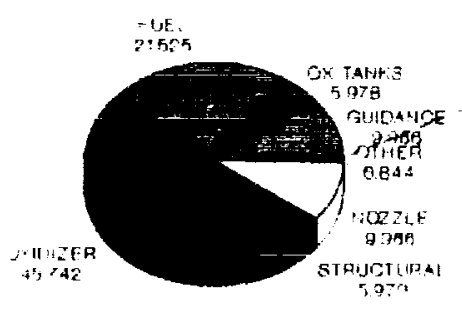

Case 2b. All-earth-carried $\mathrm{CH}_{4} / \mathrm{H}_{2} \mathrm{O}_{2}$ (Mass at LEO $=43.9$ tonnes)
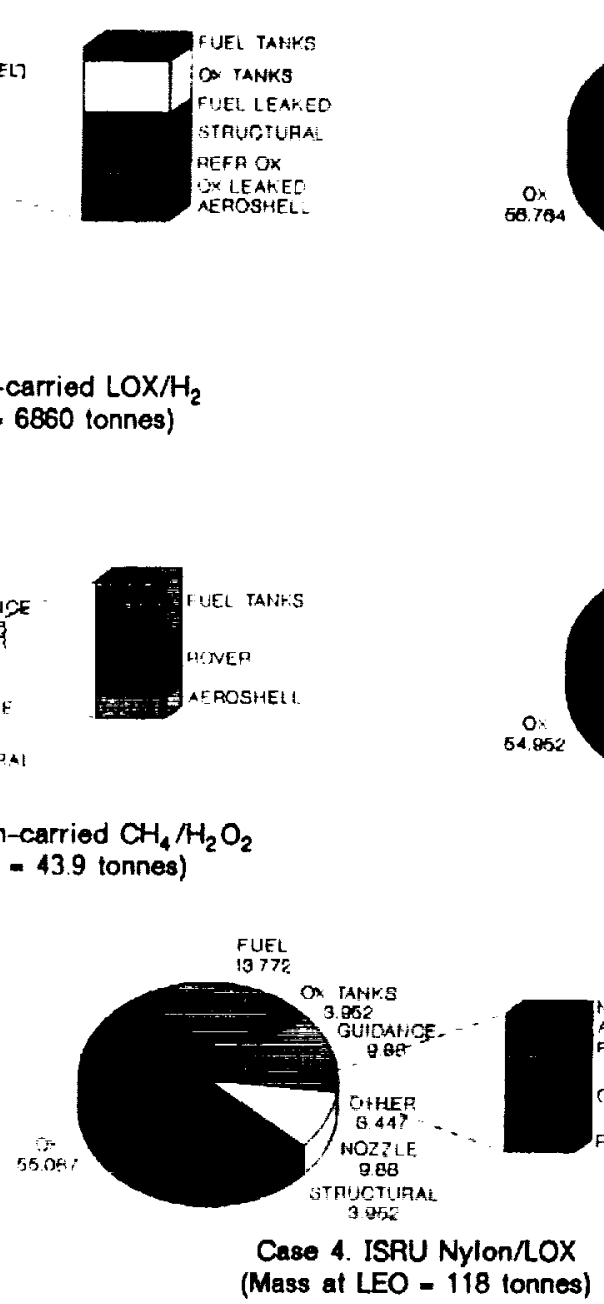

Figure 4.4 Mission mass summaries.

\section{ORIGINAL PAGE IS OF POOR QUNUTY}

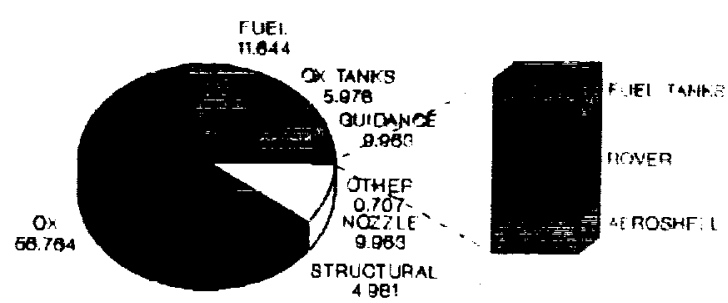

Case 2a. All-earth-carried $\mathrm{CH}_{4} / \mathrm{N}_{2} \mathrm{O}_{4}$ (Mass at LEO $=40.5$ tonnes)

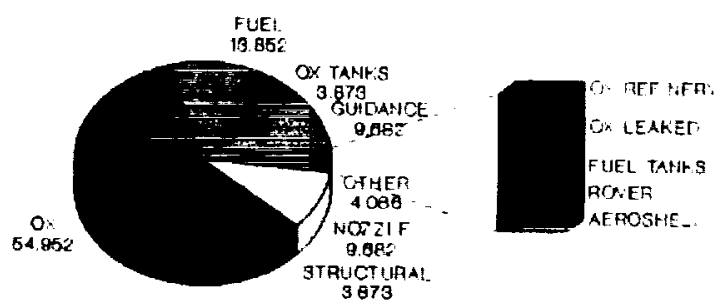

Case 3. Earth-carried $\mathrm{CH}_{4}$ nOX (ISRU) (Mass at LEO - 11.0 tonnes)

convection and solar radiation. The $\mathrm{LH}_{2}$ needs refrigeration always. During space travel, it is assumed that the infinite heat sink (space) can be used to keep the LOX cool and that no active refrigeration is used. Further innovations may alter some of these numbers. The rest of the results are straightforward solutions to the governing equations.

\section{Preliminary Findings}

As was expected (Ramohalli et al. 1987a, 1987b), the LOX/H $\mathrm{H}_{2}$ case is not the best for this mission. In order of merit, the options should be:

1. ISRU LOX/Earth-transported $\mathrm{CH}_{4}$

2. All-Earth-transported $\mathrm{H}_{2} \mathrm{O}_{2} / \mathrm{CH}_{4}$

3. All-Earth-transported $\mathrm{N}_{2} \mathrm{O}_{4} / \mathrm{CH}_{4}$

4. Spent case as fuel/ISRU LOX

5. All-Earth-transported $\mathrm{LOX} / \mathrm{H}_{2}$ 


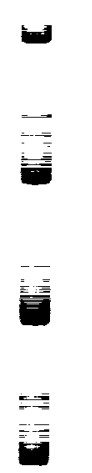




\section{Discussion}

The preliminary results of these studies indicate two important facts. One is that it is feasible to attempt a figure-of-merit approach to space missions that utilize extraterrestrial resources. The other is that the ISRU plant (hardware, equipment, controls, ...) per se is not very taxing in terms of mass penalties, but the refrigeration needs of the liquid oxidizer (LOX) produced extraterrestrially can impose a very heavy penalty on the entire mission. Nevertheless, the decisive advantages of the ISRU mission are brought out, once again, in relation to all-Earth-transported resources. However, the demands of refrigeration prompts one to seriously consider the prospects for non-cryogenically storing the valuable oxidizer produced extraterrestrially. The production itself is quite simple, if not easy, through the use of modern high-tech zirconia oxygen cells. The necessary duration of 9-12 months on Mars, dictated by the orbital mechanics of minimum energy transfers of interplanetary travel, provides us with the important opportunity of carrying a small oxidizer plant that has a small output per hour, but produces a large quantity over the 9-12 months. The main issue is to find a means of economically storing the oxidizer for that length of time.

In this regard, one scheme that may seem very radical is to think of storing the oxidizer as a solid. Many powerful oxidizers are solids, and many of these possess a fairly low (negative) enthalpy of formation, $\Delta h_{f}^{0}$. A ready example is ammonium perchlorate that has been extensively used as an oxidizer in rocket propellant over a wide temperature range. While we are not suggesting making AP on Mars, the concept of a solid oxidizer, such as ammonium nitrate, may be worth some consideration. Nitrogen is found in the Martian atmosphere, and there are indications that the polar regions of Mars may have as much as $10-14 \%$ nitrogen in the atmosphere because of some differential diffusional effects [Source: Prof. John Lewis, University of Arizona]. The point to note is that some innovation in storing and end-use of the extraterrestrially produced oxidizer may make ISRU schemes orders-of-magnitude better than they are now through the elimination of refrigeration. On other planets and moons, where very low-temperature (< melting point of oxygen) regions are present, the concept of oxygen bricks has been suggested by James Burke. Some work on these schemes is proceeding at the UA NASA Space Engineering Research Center at the University of Arizona.

\section{Future Work}

Further study will include variations in the overall mission and additional testing of the propellant options. The MSR mission needs testing for an orbiting craft left in MPO, where the sample could be transferred from the MAV and propelled on towards LEO. 
Other options would be to investigate how ISRU could be used to enhance missions to Phobos and Deimos. Other possibilities include long-term refueling stations. Propellant selection will prove vital to future analyses. Refrigeration requirements have proved to be a dominant factor in the analysis. In this light, the use of solids must also be evaluated. The mission requirements and propellant selection will determine the role of ISRU.

\section{Summary}

The approach to extraterrestrial resource utilization through the concept of an overall mission figure-of-merit leads to more realistic designs than through individual component performance maximizations. Simple examples show that the much studied LOX $/ \mathrm{H}_{2}$ propulsion system may be capable of specific impulses in the 460 to $462 \mathrm{sec}$ range, but the necessary refrigeration systems, leak-proofing, extended storage, cryogenic handling, pumping, and safety hazards all translate into excessive mass. This mass increase can easily exceed the mass ratio advantage gained through the superb specific impulse. Generalizing these thoughts, several variations were considered. It was shown that some of the less spectacular components can add up to a better system, overall. Conservative assumptions were made where specific numbers were not available. The actual numbers, although critically important for the final mission, are not critical for the purpose of this paper, which is advancing a new concept of extraterrestrial resource utilization rather than precise delineation of mission design numbers. A popular spreadsheet was used to demonstrate quantitatively the interrelationships among the various parameters. The approach is flexible. A specific MSR mission was considered to illustrate the approach at this time. It is easy to consider more involved Mars missions, such as those described by Nock and Friedlander (1987), as the mission component, instead of the very simple mission mechanics we have considered here to develop the FoM concept. Some of the unconventional propellant combinations were compared with the all-Earth-transported $\mathrm{LOX} / \mathrm{H}_{2}$ system. In summary, this new approach using a figure-of-merit may be more revealing, helpful, and meaningful than the conventional approaches of individual optimizations. An FoM approach may well become the key to economical utilizations of extraterrestrial resources to the benefit of all mankind.

\section{Acknowledgments}

The authors are grateful to Steve Hartman for support and the Advisory Committee of the Center for constructive criticisms on an earlier version of this study. 


\section{References}

Ash, R. L., et al., 1978, "Feasibility of Rocket Propellant Production on Mars," Acta Astronautica, Vol. 5, pp. 705-734.

Ash, R. L., et al., 1982, "Autonomous Oxygen Production for a Mars Return Vehicle," IAF (Paris) Paper 82-210.

Ash, R. L. and Cuda, V., Jr., 1984, "Technology Status Report for In Situ Propellant Production," Report to Jet Propulsion Lab., Pasadena, California.

Bursard, R. W. and deLauer, R. D., 1965, Fundamentals of Nuclear Flight, $2^{\text {nd }}$ Ed., McGraw Hill, New York, pp. 40-44.

Carroll, W. F. (Ed.), 1983, "Research on the Use of Space Resources, JPL Publication 83-86.

French, J. R., et al., 1985, "Mars Sample Return Options," Aerospace America, Vol. 23, pp. 50-53.

Frisbee, R. H. and Jones, R. M., 1983, "An Analysis of Propulsion Options for Transport of Lunar Materials to Earth Orbit," AIAA Paper 83-1344.

Irving, J. H. and Blum, E. K., 1959, "Comparative Performance of Ballistic and LowThrust Vehicles for Flight to Mars," pages 191-218 in Vistas in Astronautics, Vol. II (Alperin and Gregory, eds.), Pergamon Press, New York.

Nock, K. T. and Friedlander, A. L., 1987, "Elements of a Mars Transportation System," Acta Astronautica, Vol. 15, pp. 505-522.

(Presidential) National Commission on Space, Pioneering the Space Frontier, Bantam Books, New York, 1986.

Ramohalli, K. N., et al., 1987a, "Novel Extraterrestrial Processing for Space Propulsion, Acta Astronautica, Vol. 15, pp. 259-273.

Ramohalli, K. N., et al., 1987b, "Some Aspects of Space Propulsion With Extraterrestrial Processing," AIAA J. Spacecraft \& Rockets, Vol. 24, pp. 236-244.

Ramohalli, K. N., et al., 1989, "Recent Concepts in Missions to Mars--Extraterrestrial Processing," AIAA J. Propulsion \& Power, Vol. 5, p.p. 181-187.

Rascon, M., 1989, "Performance Calculations for Unconventional Propellants and Combinations," M.S. Thesis, University of Arizona, Tucson. 